\title{
Decoherence and thermalization dynamics of a quantum oscillator
}

\author{
V V Dodonov*†, S S Mizrahi ${ }^{\ddagger}$ and A L de Souza Silva ${ }^{\S}$ \\ Departamento de Física, Universidade Federal de São Carlos, \\ Via Washington Luiz, km 235, 13565-905 São Carlos, SP, Brasil
}

\begin{abstract}
We introduce the quantitative measures characterizing the rates of decoherence and thermalization of quantum systems. We study the time evolution of these measures in the case of a quantum harmonic oscillator whose relaxation is described in the framework of the standard master equation, for various initial states (coherent, 'cat', squeezed and number). We establish the conditions under which the true decoherence measure can be approximated by the linear entropy $1-\operatorname{Tr} \hat{\rho}^{2}$. We show that at low temperatures and for highly excited initial states the decoherence process consists of three distinct stages with quite different time scales. In particular, the 'cat' states preserve $50 \%$ of the initial coherence for a long time interval which increases logarithmically with increase of the initial energy.
\end{abstract}

Keywords: decoherence; relaxation; master equation; quantum oscillator

* On leave from Lebedev Physical Institute and Moscow Institute of Physics and Technology, Russia

$\dagger$ e-mail: vdodonov@power.ufscar.br

$\ddagger$-mail: salomon@power.ufscar.br

$\S$ On leave from Universidade Federal de Rondônia, Brazil

๑e-mail: palus@iris.ufscar.br 


\section{Introduction}

Recently, a significant interest to the decoherence processes in quantum mechanics is observed, in particular, due to the problem of stability of quantum superpositions (frequently modeled by some kinds of the 'Schrödinger cats' [1]-[5]) under the influence of the environment [6]- [15]. However, despite that the qualitative picture of the phenomenon seems more or less clear, there are no unique quantitative measures of the rate of decoherence or the rate of thermalization. The decoherence implies a degradation of the quantum interference effects (manifesting themselves in the existence of quantum superpositions) due to the interactions with the 'external world'. Since these effects are inherent to the pure quantum states, while they disappear in quantum mixtures, it seems natural, on the face of it, to identify the 'degree of decoherence' with the degree of 'impurity' of the quantum state, expressed in terms of the 'canonical entropy' [16] $S=-\operatorname{Tr}(\hat{\rho} \ln \hat{\rho})$ or in terms of the 'linear entropy' [11, 14, 17 $s=1-\operatorname{Tr} \hat{\rho}^{2}$, which is more simple for calculations. However, a deeper analysis shows that such an identification leads to certain difficulties, especially in the low temperature case.

Indeed, let us consider the evolution of an initial pure $(s(0)=S(0)=0)$ quantum state due to a weak interaction with a large reservoir at low temperature. For $t>0, s(t)$ and $S(t)$ assume positive values, so the rate of increase of $s(t)$ or $S(t)$ at $t \rightarrow 0$ can provide us some hints to the time scale of the initial phase of the decoherence process [11, 14]. However, tracing the evolution of the entropies for the long time interval, we discover that for a small enough temperature of the environment, the entropies, after reaching some maxima, finally decrease to very small values which tend to zero when $T \rightarrow 0$ (because the stationary thermal mixed state is very close in this case to the ground pure state). Then, identifying the measure of quantum impurity with the measure of decoherence, one should accept a strange result that the degree of decoherence of the final equilibrium state is almost the same (close to zero) as it was initially, despite that the thermal states are usually believed to be the most 'incoherent'

This example shows that at low temperatures the entropies can serve as the measures of decoherence only at the initial stage of the decoherence process. Thus, several questions arise. The first one: is it possible to find some other measures which could be used in the whole interval $0 \leq t<\infty$ and for any temperature of the environment? Another question is: under which conditions (at which time scale) the usage of the entropies as the measures of the decoherence can be justified?

In the present paper, we answer both questions, introducing the new parameter $\mathcal{C}$ whose connection with the degree of coherence is indubitable (see section 2). This parameter equals one for pure quantum states and 0 for the thermodynamical equilibrium states, for any temperature $T>0$. In section 3 we introduce another parameter $\mathcal{D}$, which can be considered as the 'measure of thermalization', since it equals zero for any pure state and 1 for the thermodynamically equilibrium state of any quantum system with an equidistant energy spectrum, for any temperature $T>0$.

Following the time evolution of the parameters $\mathcal{C}$ and $\mathcal{D}$ in the process of the thermal relaxation of various initial states (Fock's, coherent, squeezed, 'cat') of the harmonic oscillator, described in the framework of the 'standard master equation' (sections 1 and 5), we find the conditions, under which the linear entropy can serve as a reasonable 'measure of decoherence'. Moreover, in section 6 we demonstrate that the decoherence of highly excited initial states at low temperatures goes through three distinct stages, characterized not by some unique 'decoherence 
time', but at least by two times with quite different dependences on the initial energy and the temperature.

The first time $t_{1}$ (which is usually identified with the time of decoherence) is, roughly speaking, inversly proportional to the product of the energy of quantum fluctuations by the number of photons per mode of the reservoir. During this short time interval the parameter $\mathcal{C}$ rapidly decreases from 1 to some finite value which depends on the initial state. Then $\mathcal{C}(t)$ remains at a more or less constant level (for the 'cat' states) or even can increase with time (for the 'squeezed' states). And only after the 'ultimate decoherence time' $t_{d}$, which increases (logarithmically) with the increase of the initial energy, the coherence coefficient goes monotonously to the final zero value.

The evolution of the 'thermalization parameter' $\mathcal{D}(t)$ is similar to certain extent to the behaviour of $1-\mathcal{C}(t)$ : the initial rapid increase from zero to some intermediate value, then some stabilization or even decrease, and the final rapid transition to the equilibrium unit value after the 'thermalization time' $t_{T}$, which also depends logarithmically on the initial energy. The difference between $t_{d}$ and $t_{T}$ consists in their temperature dependences: $t_{d}$ has a finite limit value when the temperature $T$ tends to zero, whereas $t_{T}$ is inversly proportional to the absolute temperature at $T \rightarrow 0$, in accordance with the third law of thermodynamics (the inattainability of the absolute zero of temperature implies that the rate of the relaxation processes must go to zero as $T \rightarrow 0)$.

\section{The measure of 'coherence'}

The controversies of the identification the decoherence measure with the von Neumann' or linear entropies take their origin in the invariance of these entropies with respect to the choice of the basis in the Hilbert space: the entropies do not distinguish the equilibrium state (or other stationary states in the case of 'colored' or 'squeezed' reservoirs) from any other mixed one. But such a symmetry with respect to the choice of the basis in the Hilbert space is obviously broken in the relaxation processses, when all possible initial states tend to the unique equilibrium state, whose density matrix is diagonal in the distinct basis formed by the energy eigenstate of the Hamiltonian (or by some other distinct basis in the case of more sophisticated artificial reservoirs considered recently). Thus it seems natural to suppose that the measure of decoherence must depend explicitly on this distinct basis $|n\rangle\langle n|$ (the concept of the broken symmetry of the Hilbert space was used as a basis for introducing the polarized distance between different quantum states in [18]). In some special cases, when it is known exactly to which specific family of quantum states (e.g., coherent state, even/odd coherent state, etc.) the initial quantum state belongs, the prefered basis may be different from the energy one, so that some special approaches can be used, as well. We shall discuss such a situation later on. However, in the generic case, when the type of the quantum state is not known beforehand, the only available information is contained in the set of matrix elements of the statistical operator with respect to the physically distinguished energy basis: $\hat{\rho}(t)=\sum_{m n} \rho_{m n}|m\rangle\langle n|$. Therefore, it seems reasonable to define the measure of coherence in terms of the coefficients $\rho_{m n}$. Since

the decoherence is usually identified with the disappearence of the off-diagonal elements of the 
density matrix $\left\|\rho_{m n}\right\|$, it is natural to define the normalized measure of coherence as

$$
\mathcal{C}(t)=\sum_{m \neq n}\left|\rho_{m n}\right|^{2}(t) / \sum_{m \neq n}\left|\rho_{m n}\right|^{2}(0)
$$

Then $\mathcal{C}(0)=1$, while $\mathcal{C} \equiv 0$ for any 'completely incoherent' state without off-diagonal matrix elements in the energy basis (provided initially at least one off-diagonal element was different from zero). Introducing the 'diagonal part' of the operator $\hat{\rho}$

$$
\hat{\rho}_{d}=\sum_{n} p_{n}|n\rangle\langle n|, \quad p_{n} \equiv\langle n|\hat{\rho}| n\rangle
$$

and taking into account the property $\operatorname{Tr}\left(\hat{\rho} \hat{\rho}_{d}\right)=\operatorname{Tr}\left(\hat{\rho}_{d}^{2}\right)$ one can rewrite (1) in the form

$$
\begin{gathered}
\mathcal{C}=\frac{\operatorname{Tr}\left[\hat{\rho}(t)-\hat{\rho}_{d}(t)\right]^{2}}{\operatorname{Tr}\left[\hat{\rho}(0)-\hat{\rho}_{d}(0)\right]^{2}} \equiv \frac{\mu(t)-\lambda(t)}{\mu(0)-\lambda(0)} \\
\mu \equiv \operatorname{Tr} \hat{\rho}^{2}, \quad \lambda \equiv \operatorname{Tr} \hat{\rho}_{d}^{2}=\sum_{n} p_{n}^{2}
\end{gathered}
$$

We shall call $\mu$ the 'total purity' and $\lambda$ the 'diagonal purity'. In many cases of practical interest both 'purities' can be calculated rather easily. For example, if one knows the Wigner function [19, 20] (we assume $\hbar \equiv 1$ )

$$
\begin{gathered}
W(q, p)=\int d v e^{i p v}\left\langle q-\frac{v}{2}|\hat{\rho}| q+\frac{v}{2}\right\rangle \\
\operatorname{Tr} \hat{\rho}=\int W(q, p) d q d p /(2 \pi)=1
\end{gathered}
$$

then

$$
\mu=\int W^{2}(q, p) d q d p /(2 \pi)
$$

As to the 'diagonal purity', it can be calculated either by means of a direct summation of the series in (4), or, equivalently, it can be expressed as the integral

$$
\lambda(t)=\int_{0}^{2 \pi} \frac{d \varphi}{2 \pi}\left|G\left(e^{i \varphi} ; t\right)\right|^{2}
$$

where

$$
G(z ; t) \equiv \sum_{n=0}^{\infty} p_{n}(t) z^{n}
$$

is the diagonal generating function. Below we use both methods.

\section{The measure of thermalization}

A qualitative measure of thermalization can be introduced in the following way. The analysis of the low temperature behaviour of the entropies shows that the troubles mentioned in the introduction arise due to the double nature of the ground state, described by the density operator $\hat{\rho}_{0} \equiv|0\rangle\langle 0|$. On one hand, this state is pure, with $\operatorname{Tr} \hat{\rho}_{0}^{2}=1$. On the other hand, it is 
the limit of the equilibrium states, which are conceived to be completely decoherent. Therefore it seems reasonable to exclude the state $\hat{\rho}_{0}$ in some way. One of the possibilities is to take a simple expression for the linear entropy and to divide it by a proper time-dependent factor which would ensure a nonzero limit at $t \rightarrow \infty$. This goal can be achieved, for instance, if one chooses as the normalizing factor the Hilbert-Schmidt distance between the states $\hat{\rho}(t)$ and $\hat{\rho}_{0}$. If the system under study has a finite number of energy levels (e.g., spin systems), then there are some grounds to treat the state with the maximal energy $\hat{\rho}_{f} \equiv\left|E_{\max }\right\rangle\left\langle E_{\max }\right|$ on the same footing as $\hat{\rho}_{0}$. Thus we arrive at the parameter (introduced for the first time in [41], but identified erroneously with the measure of decoherence)

$$
\begin{aligned}
\mathcal{D} & =\frac{1-\operatorname{Tr} \hat{\rho}^{2}}{\left[\operatorname{Tr}\left(\hat{\rho}-\hat{\rho}_{0}\right)^{2} \operatorname{Tr}\left(\hat{\rho}-\hat{\rho}_{f}\right)^{2}\right]^{1 / 2}} \\
& =\frac{1-\mu}{\left[\left(1+\mu-p_{f}\right)\left(1+\mu-p_{0}\right)\right]^{1 / 2}}
\end{aligned}
$$

where $p_{0} \equiv \operatorname{Tr}\left(\hat{\rho} \hat{\rho}_{0}\right)=\langle 0|\hat{\rho}| 0\rangle$ is the ground state occupation probability, while $p_{f} \equiv \operatorname{Tr}\left(\hat{\rho} \hat{\rho}_{f}\right)$ is the occupation probability of the level with the maximal energy (evidently, for quantum systems with infinite dimensional Hilbert spaces, such as a harmonic oscillator, $p_{f} \equiv 0$ for any physical state possessing finite energy).

For systems with equidistant spectra, $E_{n+1}-E_{n}=\Delta E=$ const, the equilibrium occupation probabilities read

$$
p_{n}=\xi^{n}(1-\xi) /\left(1-\xi^{M}\right)
$$

where $M$ is the total number of levels, $n=0,1, \ldots, M-1$ and $\xi=\exp (-\beta \Delta E)<1$ is the Boltzmann factor. Then

$$
\begin{gathered}
\mu_{e q}=(1-\xi)\left(1+\xi^{M}\right) /\left[(1+\xi)\left(1-\xi^{M}\right)\right], \\
p_{0}^{(e q)}=(1-\xi) /\left(1-\xi^{M}\right) \\
p_{f}^{(e q)}=\xi^{M-1}(1-\xi) /\left(1-\xi^{M}\right)
\end{gathered}
$$

and we see that $\mathcal{D}_{e q} \equiv 1$ for any value $0<\xi<1$, while $\mathcal{D} \equiv 0$ for all pure states. For this reason, we may consider $\mathcal{D}$ as 'the measure of thermalization'. Note that we have an indeterminacy in (9) if $\xi=T=0$, thus the case $T=0$ must be excluded. But as we know the limit of exact zero temperature is an idealization, thus we do not have to worry on this issue.

For systems with nonequidistant spectra the value of $\mathcal{D}_{e q}$ depends on temperature, nonetheless the limits at $T \rightarrow 0$ and $T \rightarrow \infty$ still equal 1 . For $T \rightarrow \infty$ we have $p_{0}=p_{1}=\ldots=p_{f}=$ $1 / M$, consequently $\mu=M \cdot(1 / M)^{2}=1 / M$ and $\mathcal{D}=1$. In the low temperature case $T \rightarrow 0$, the equilibrium statistical operator is close to $p_{0}|0\rangle\left\langle 0\left|+p_{1}\right| 1\right\rangle\langle 1|$ with $p_{1} \ll 1$ (where $|1\rangle$ is the first excited state), while the contribution of other states can be neglected (we consider the systems with discrete energy spectra). Then $p_{0}+p_{1}=1, \mu=p_{0}^{2}+p_{1}^{2}, 1+\mu-2 p_{0}=2 p_{1}^{2}, 1+\mu-2 p_{f}=2$, and $1-\mu=2 p_{1}$ (up to higher order terms). As a result, we have $\mathcal{D}=1$ again. 


\section{Decoherence dynamics}

\subsection{Time evolution of the quantum state}

We confine ourselves to the analysis of the time dependence of the 'coherence coefficient' $\mathcal{C}$ (3) in the process of thermal relaxation of the harmonic oscillator described in the framework of the standard master equation [21, 22] (more general models were considered, e.g., in [17])

$$
\begin{aligned}
& d \hat{\rho} / d t=\gamma(1+\nu)\left(2 \hat{a} \hat{\rho} \hat{a}^{\dagger}-\hat{a}^{\dagger} \hat{a} \hat{\rho}-\hat{\rho} \hat{a}^{\dagger} \hat{a}\right) \\
& +\gamma \nu\left(2 \hat{a}^{\dagger} \hat{\rho} \hat{a}-\hat{a} \hat{a}^{\dagger} \hat{\rho}-\hat{\rho} \hat{a} \hat{a}^{\dagger}\right)-i\left[\hat{a}^{\dagger} \hat{a}, \hat{\rho}\right] .
\end{aligned}
$$

Here $\hat{a}$ and $\hat{a}^{\dagger}$ are the usual bosonic annihilation and creation operators, $\nu$ is the equilibrium mean number of quanta in the reservoir corresponding to the given mode, and $\gamma>0$ is a damping coefficient $(\hbar=\omega=1)$.

An immediate consequence of equation (10) is the universal expression for the purity loss rate in an initial pure state $\hat{\rho}^{2}=\hat{\rho}=|\psi\rangle\langle\psi|$ (cf. [11, 17, 23, 24])

$$
\left.\dot{\mu}\right|_{t=0}=\left.2 \operatorname{Tr}(\hat{\rho} \hat{\dot{\rho}})\right|_{t=0}=-4 \gamma\left[\nu+(1+2 \nu) \sigma_{a}\right],
$$

where $\sigma_{a} \equiv\left\langle\psi\left|\hat{a}^{\dagger} \hat{a}\right| \psi\right\rangle-|\langle\psi|\hat{a}| \psi\rangle|^{2}$. The 'primary' purity loss rate is minimal for the coherent states with $\sigma_{a} \equiv 0$. In the generic case it is roughly proportional to the average number of thermal photons in the reservoir and to the 'reduced' energy of quantum fluctuations in the initial pure state,

$$
\mathcal{E}_{0}=\frac{1}{2}\left[\sigma_{p}^{(0)}+\sigma_{q}^{(0)}\right] \equiv \sigma_{a}+\frac{1}{2},
$$

where $\sigma_{q}^{(0)}$ and $\sigma_{p}^{(0)}$ are the variances of the quadrature components $\hat{q}=\left(\hat{a}+\hat{a}^{\dagger}\right) / \sqrt{2}$ and $\hat{p}=i\left(\hat{a}^{\dagger}-\hat{a}\right) / \sqrt{2}$ in the initial pure state $|\psi\rangle$.

Due to equation (11) the initial evolution of the 'purity' has the linear dependence on time $\mu(t)=1-t / t_{1}+\cdots$, where the 'primary purity loss time' equals

$$
t_{1}=(4 \gamma)^{-1}\left[\nu+(1+2 \nu) \sigma_{a}\right]^{-1} .
$$

Note that some 'microscopic' models, based on an explicit coupling of the system under study with a large reservoir, result in a quadratic time dependence $\mu(t)$ at $t \rightarrow 0$ [14. This apparent contradiction is explained by the fact that the 'microscopic' models and the phenomenological master equations describe the evolution of the subsystem in different time scales. Actually, the master equation describes a 'coarse-grained' evolution averaged over many periods of the fast oscillation, so the physical meaning of the limit $t \rightarrow 0$ in the case of the master equation is quite different from the same (formally) limit in the 'microscopic' models.

To calculate the time dependence of the 'purity' $\mu$ in the whole interval $0<t<\infty$ with the aid of formula (6) we need the time dependent Wigner function $W(q, p, t)$. It obeys the Fokker-Planck equation which follows immediately from (10):

$$
\begin{aligned}
\frac{\partial W}{\partial t}= & \frac{\partial}{\partial q}([\gamma q-p] W)+\frac{\partial}{\partial p}([\gamma p+q] W) \\
& +\gamma\left(\nu+\frac{1}{2}\right)\left(\frac{\partial^{2} W}{\partial q^{2}}+\frac{\partial^{2} W}{\partial p^{2}}\right) .
\end{aligned}
$$


The solution to equation (14) can be written as

$$
W(q, p ; t)=\int \mathcal{K}\left(q, p ; t \mid q^{\prime}, p^{\prime}, 0\right) W\left(q^{\prime}, p^{\prime} ; 0\right) d q^{\prime} d p^{\prime}
$$

The propagator $\mathcal{K}\left(q, p ; t \mid q^{\prime}, p^{\prime}, 0\right)$ was calculated by means of different methods in [25, 26, 27, 28]; the explicit expressions in the case of most general multidimensional time-dependent quadratic operator (with respect to $q, p, \partial / \partial q, \partial / \partial p$ ) in the right-hand side of the Fokker-Planck equation were given in [29, 30. In the case involved the general form found in [29] is reduced to (see appendix)

$$
\begin{aligned}
& \mathcal{K}\left(q, p ; t \mid q^{\prime}, p^{\prime}, 0\right)=\left(2 \pi \sigma_{*} u\right)^{-1} \exp \left\{-\left(2 \sigma_{*} u\right)^{-1}\left[q_{t}^{2}+p_{t}^{2}\right.\right. \\
& \left.\left.+e^{-2 \gamma t}\left(q^{\prime 2}+p^{\prime 2}\right)-2 e^{-\gamma t}\left(q^{\prime} q_{t}+p^{\prime} p_{t}\right)\right]\right\}
\end{aligned}
$$

where $\sigma_{*} \equiv \nu+\frac{1}{2}$,

$$
q_{t}=q \cos t-p \sin t, \quad p_{t}=p \cos t+q \sin t,
$$

and the 'compact time' $u$ is given by

$$
u(t) \equiv 1-e^{-2 \gamma t}
$$

The consequence of the master equation (10) is the closed set of equations for the diagonal elements of the density matrix in the Fock (energy) basis

$$
\begin{aligned}
\dot{p}_{n}= & 2 \gamma(1+\nu)\left[(n+1) p_{n+1}-n p_{n}\right] \\
& +2 \gamma \nu\left[n p_{n-1}-(n+1) p_{n}\right] .
\end{aligned}
$$

These equations, in turn, are equivalent to the simple first order partial differential equation for the diagonal generating function (8)

$$
\frac{\partial G}{\partial t}=2 \gamma(1-z)[1+\nu(1-z)] \frac{\partial G}{\partial z}-2 \gamma \nu(1-z) G
$$

The solution to (19) reads [31, 32, 33]

$$
G(z, u)=\frac{1}{1+\nu u(1-z)} G_{0}\left(\frac{z+u(1+\nu)(1-z)}{1+\nu u(1-z)}\right)
$$

where $G_{0}(z) \equiv G(z, 0)$. Putting $z=1$ in (20) we verify the normalization condition $G(1, t) \equiv 1$.

\subsection{Initial coherent states}

As the first example we consider the evolution of the initial coherent state $|\alpha\rangle, \alpha \equiv \sqrt{a} \exp (i \phi)$. Applying the propagator (16) to the initial Wigner function

$$
W^{(c o h)}(0)=2 \exp \left[-(q-\sqrt{2 a} \cos \phi)^{2}-(p-\sqrt{2 a} \sin \phi)^{2}\right]
$$


we obtain

$$
\begin{aligned}
W^{(c o h)}(q, p, t)= & 2 \xi_{\nu} \exp \left\{-\xi_{\nu}\left(\left[q-\sqrt{2 a} e^{-\gamma t} \cos (\phi-t)\right]^{2}\right.\right. \\
& \left.\left.-\left[p-\sqrt{2 a} e^{-\gamma t} \sin (\phi-t)\right]^{2}\right)\right\}
\end{aligned}
$$

where

$$
\xi_{\nu}(u) \equiv(1+2 u \nu)^{-1}
$$

coincides with the 'total purity': $\mu^{(\mathrm{coh})}=\xi_{\nu}(u)$.

To calculate the 'diagonal purity' we use the explicit expression for the time-dependent diagonal matrix elements in terms of the Laguerre polynomials [31, 33]

$$
p_{n}^{(c o h)}=\frac{(u \nu)^{n}}{(1+u \nu)^{n+1}} \exp \left[\frac{a(u-1)}{1+u \nu}\right] L_{n}\left[\frac{a(u-1)}{u \nu(1+u \nu)}\right]
$$

Then the sum in (4) is reduced to the known series [34]

$$
\sum_{n=0}^{\infty} L_{n}(x) L_{n}(y) z^{n}=(1-z)^{-1} \exp \left[z \frac{x+y}{z-1}\right] I_{0}\left[2 \frac{\sqrt{x y z}}{1-z}\right]
$$

$\left(I_{0}(z)\right.$ is the modified Bessel function), so we obtain

$$
\begin{gathered}
\lambda^{(c o h)}=\xi_{\nu}(u) \exp (-\eta) I_{0}(\eta), \\
\eta=2 a(1-u) \xi_{\nu}(u) .
\end{gathered}
$$

For $a \ll 1$ it is sufficient to take into account the first terms of the Taylor expansion of the function $e^{-\eta} I_{0}(\eta)$ to obtain

$$
\mathcal{C}^{(c o h)}(t) \approx \xi_{\nu}^{2}(u)(1-u)=\mu^{2}(t) e^{-2 \gamma t}
$$

In this case the time dependence of the 'purity' has a little in common with the time dependence of the 'coherence'; the same is true even for $a \sim 1$ : see figure 1 .

For the highly excited initial states with $a \gg 1$ the asymptotics of the modified Bessel function, $I_{0}(x) \approx(2 \pi x)^{-1 / 2} e^{x}$ for $x \gg 1$, yields

$$
\mathcal{C}^{(c o h)}(t) \approx \mu^{(c o h)}(t)\left[1-\frac{1}{2 \sqrt{a \pi}}\left(\sqrt{1+2 u \nu} e^{\gamma t}-1\right)\right] .
$$

In this case, the contribution of the diagonal elements to the total purity is small compared with the contribution of the off-diagonal terms, therefore the sum over $m \neq n$ in (11) can be replaced by the sum over all values of $m$ and $n$, and the correlation coefficient can be approximated by the purity $\mu$. This is just the case considered in most of the papers devoted to the decoherence of initial 'macroscopic' quantum states. However, $\mathcal{C} \approx \mu$ only under the condition $\lambda \ll \mu$. Consequently, the linear entropy $1-\mu$ can be considered as a measure of decoherence only for $a \gg 1$, and under the additional restriction $a \xi_{\nu}(u) \exp (-2 \gamma t) \gg 1$, i.e., at the time scale

$$
t \ll t_{*} \approx(2 \gamma)^{-1} \ln [(a+2 \nu) /(1+2 \nu)] .
$$


For $t \geq t_{*}$ the identification of the 'purity' with the 'coherence' leads to incorrect results as mentioned in the introduction.

The 'final decoherence time' $t_{d}$ can be defined by means of the equation

$$
\mathcal{C}\left(t_{d}\right)=\beta \mu_{e q}
$$

where $\beta<1$ is some small number whose choice is a matter of convention (say, $\beta=0.1$ ), and $\mu_{e q}=(1+2 \nu)^{-1}$ is the equilibrium value of the 'purity' (we take into account that $\mathcal{C}$ is proportional to $\xi_{\nu}=\mu^{(c o h)}$, according to equation (25)). To solve equation (29) for sufficiently small $\beta$ we can use the asymptotical form of the coherence function at $\gamma t \gg 1$ (when $u \approx 1$ )

$$
\mathcal{C}^{(c o h)}(t) \approx 2 a(1+2 \nu)^{-2} e^{-2 \gamma t}, \quad a \gg 1, \quad \gamma t \gg 1 .
$$

Thus we obtain the estimation

$$
t_{d}^{(c o h)} \approx(2 \gamma)^{-1} \ln \left[2 a \mu_{e q} / \beta\right]
$$

which holds for $a \mu_{e q} \gg 1$. The evolution of the 'total purity' and the 'coherence coefficient' for highly excited initial coherent states at different temperatures is shown in figure $\mathrm{Q}$.

\subsection{Initial 'cat' states}

Now let us consider the family of the initial 'Schrödinger cat' states [4, 5, 9]

$$
\begin{gathered}
|\alpha ; \varphi\rangle=\mathcal{N}\left(|\alpha\rangle+e^{i \varphi}|-\alpha\rangle\right) \\
\mathcal{N}=(2[1+\cos \varphi \exp (-2 a)])^{-1 / 2}, \quad a \equiv|\alpha|^{2} .
\end{gathered}
$$

The special cases of this family are even $(\varphi=0)$ and odd $(\varphi=\pi)$ coherent states [2], and the Yurke-Stoler states $(\varphi=\pi / 2)$ [3]. The Wigner function of the state (31) reads (hereafter we assume $\alpha=\sqrt{a}$ to be real)

$$
\begin{aligned}
& W^{(c a t)}(q, p ; 0)=4 \mathcal{N}^{2} \exp \left(-q^{2}-p^{2}\right) \\
& \times\left[e^{-2 a} \cosh (\sqrt{8 a} q)+\cos (\sqrt{8 a} p+\varphi)\right] .
\end{aligned}
$$

Applying the propagator (16) to this function we obtain (see also [10])

$$
\begin{aligned}
& W^{(c a t)}(q, p ; t)=4 \mathcal{N}^{2} \xi_{\nu} \exp \left[-\xi_{\nu}\left(q^{2}+p^{2}\right)\right] \\
& \times\left\{\exp \left[-2 a(1-u) \xi_{\nu}\right] \cosh \left[\xi_{\nu} \sqrt{8 a(1-u)} q_{t}\right]\right. \\
& \left.+\exp \left[-2 a(1+2 \nu) u \xi_{\nu}\right] \cos \left[\xi_{\nu} \sqrt{8 a(1-u)} p_{t}+\varphi\right]\right\}
\end{aligned}
$$

where the function $\xi_{\nu}(u)$ was defined in equation (22). Calculating the integral in (6) we find the 'total purity'

$$
\begin{aligned}
& \mu=2 \mathcal{N}^{4} \xi_{\nu}(u)\left[1+4 \cos \varphi e^{-2 a}+\cos (2 \varphi) e^{-4 a}\right. \\
& \left.+\exp \left[-4 a(1-u) \xi_{\nu}\right]+\exp \left[-4 a u(1+2 \nu) \xi_{\nu}\right]\right]
\end{aligned}
$$


The photon distribution function can be written as

$$
p_{n}^{(c a t)}=2 \mathcal{N}^{2}\left[p_{n}^{(c o h)}(a)+\cos \varphi e^{-2 a} p_{n}^{(c o h)}(-a)\right],
$$

where $p_{n}^{(\text {coh })}(a)$ is given by (23). Calculating again the sum $\sum p_{n}^{2}$ with the aid of formula (24) we find

$$
\begin{aligned}
& \lambda^{(c a t)}=4 \mathcal{N}^{4} \xi_{\nu}(u)\left\{I_{0}(\eta)\left[e^{-\eta}+\cos ^{2} \varphi e^{\eta-4 a}\right]\right. \\
& \left.+2 \cos \varphi e^{-2 a} J_{0}(\eta)\right\}
\end{aligned}
$$

where $J_{0}(z)$ means the usual Bessel function, and $\eta$ was defined in equation (26). If $a \ll 1$, then we have $\mu \approx \xi_{\nu}(u)$ and the same formula (27) for $\lambda$.

For highly excited ('macroscopic': $a \gg 1$ ) initial cat states the phase $\varphi$ becomes unimportant, and $\mathcal{N}^{2} \approx 1 / 2$. Until $a(1-u) \equiv a \exp (-2 \gamma t) \gg 1$, the 'diagonal purity' is small, similarly to the case of coherent states: $\lambda^{(\text {cat })} \sim \exp (\gamma t) / \sqrt{a}$. Then the coherence coefficient can be replaced by the 'total purity' $\mu$, which rapidly decreases from 1 to the value $\frac{1}{2} \xi_{\nu}(u)$ :

$$
\mu \approx \frac{1}{2} \xi_{\nu}(u)\left(1+\exp \left[-4 a u(1+2 \nu) \xi_{\nu}(u)\right]\right),
$$

staying at this level until $a(1-u)$ becomes smaller than 1 . In particular, in the low temperature case $\nu \ll 1$ we observe the 'plateau' $\mu \approx \mathcal{C} \approx \frac{1}{2}$ : see figure (3).

At the final stage of the evolution $\mu$ goes to the equilibrium value $\mu_{e q}=(1+2 \nu)^{-1}$ as

$$
\mu \approx \frac{1}{2} \xi_{\nu}(u)\left(1+\exp \left[-4 a(1-u) \xi_{\nu}(u)\right]\right),
$$

but now it becomes compatible with the 'diagonal purity' $\lambda \approx \xi_{\nu}(u) \exp \left[-2 a(1-u) \xi_{\nu}(u)\right]$, so

$$
\mathcal{C}^{(c a t)} \approx \frac{1}{2} \xi_{\nu}(u)\left(1-\exp \left[-2 a(1-u) \xi_{\nu}(u)\right]\right)^{2} .
$$

For $a(1-u) \ll 1$ we have $\mathcal{C}^{(c a t)} \approx 2 a^{2} \mu_{e q}^{3} \exp (-4 \gamma t)$. Then equation (29) yields the 'ultimate decoherence time' $t_{d}^{(c a t)} \approx(2 \gamma)^{-1} \ln \left[a \mu_{e q} \sqrt{2 / \beta}\right]$ (if $a \mu_{e q} \gg 1$ ), which is only slightly less than the similar time $t_{d}^{(\text {coh })}(30)$.

\subsection{Decoherence in the accompanying basis}

In the special case of the 'cat' states there exists another natural choice of the 'diagonal' part of the statistical operator, different from (2). Namely, one can define the 'accompanying' diagonal operator as $\hat{\rho}_{\text {mix }}=\frac{1}{2}(|\alpha\rangle\langle\alpha|+|-\alpha\rangle\langle-\alpha|)$. The Wigner function of this quantum mixture is the sum of two coherent Wigner functions (21) with opposite values of parameter $\alpha$ :

$$
\begin{aligned}
& W_{m i x}(q, p ; t)=\frac{1}{2}\left[W_{\alpha}+W_{-\alpha}\right] \\
& =2 \xi_{\nu}(u) \exp \left\{-\xi_{\nu}(u)\left[q^{2}+p^{2}+2 a(1-u)\right]\right\} \\
& \times \cosh \left[\xi_{\nu}(u) \sqrt{8 a(1-u)} q_{t}\right] .
\end{aligned}
$$


Then the 'accompanying' (normalized) coherence coefficient can be defined as

$$
\begin{aligned}
& \mathcal{F}=\int\left[W^{(c a t)}(t)-W_{\text {mix }}(t)\right]^{2} d q d p \\
& \times\left\{\int\left[W^{(c a t)}(0)-W_{\text {mix }}(0)\right]^{2} d q d p\right\}^{-1}
\end{aligned}
$$

Performing the calculations we obtain

$$
\begin{aligned}
& \mathcal{F}=\frac{\xi_{\nu}(u)\left(1-\exp \left[-4 a(1-u) \xi_{\nu}\right]\right)}{\left(1-\cos ^{2} \varphi e^{-4 a}\right)\left(1-e^{-4 a}\right)} \\
& \times\left(\exp \left[-4 a u(1+2 \nu) \xi_{\nu}\right]-\cos ^{2} \varphi e^{-4 a}\right)
\end{aligned}
$$

The time evolution of this parameter is essentially different from the evolution of the coefficient $\mathcal{C}$ : see figure (4). This is not surprising, since the phenomena observed from the moving ('accompanying') frame of reference in many cases look very different, compared with their appearance in the fixed frame.

\subsection{Initial squeezed states}

Now let us consider the states possessing the Gaussian Wigner functions [24, 29, 35, 36]

$$
\begin{aligned}
& W(q, p)=d^{-1 / 2} \exp \left\{-\frac{1}{2 d}\left[\sigma_{p}(q-\bar{q})^{2}\right.\right. \\
& \left.\left.-2 \sigma_{q p}(q-\bar{q})(p-\bar{p})+\sigma_{q}(p-\bar{p})^{2}\right]\right\},
\end{aligned}
$$

where $\sigma_{q}, \sigma_{p}$ and $\sigma_{q p}=\sigma_{p q}$ are the (co)variances of the quadrature components, whereas $\bar{q}$ and $\bar{p}$ stand for the average values of these quadratures. The parameter $d \equiv \sigma_{p} \sigma_{q}-\sigma_{p q}^{2}$ must satisfy the Schrödinger-Robertson uncertainty relation [30, 37, 38] $d \geq 1 / 4$. It is related to the 'purity' of the state as $\mu=(4 d)^{-1 / 2}$. In the thermal state, $\sigma_{q}^{(e q)}=\sigma_{p}^{(e q)}=\sigma_{*} \equiv \frac{1}{2}+\nu, \sigma_{p q}=\bar{q}=\bar{p}=0$.

The evolution of the five parameters determining the Gaussian state is governed by the set of equations following from the master equation (10)

$$
\begin{aligned}
d \bar{q} / d t & =\bar{p}-\gamma \bar{q}, \quad d \bar{p} / d t=-\bar{q}-\gamma \bar{p} \\
\dot{\sigma}_{q} & =2 \sigma_{q p}-2 \gamma \sigma_{q}+\gamma(1+2 \nu) \\
\dot{\sigma}_{p} & =-2 \sigma_{q p}-2 \gamma \sigma_{p}+\gamma(1+2 \nu) \\
\dot{\sigma}_{q p} & =\sigma_{p}-\sigma_{q}-2 \gamma \sigma_{q p}
\end{aligned}
$$

The solutions read [29]

$$
\begin{aligned}
& \bar{q}(t)=e^{-\gamma t}\left[\bar{q}_{0} \cos t+\bar{p}_{0} \sin t\right] \\
& \bar{p}(t)=e^{-\gamma t}\left[\bar{p}_{0} \cos t-\bar{q}_{0} \sin t\right] \\
& \sigma_{q}(t)=\sigma_{*}+e^{-2 \gamma t}\left[\left(\sigma_{q}^{(0)}-\sigma_{*}\right) \cos ^{2} t\right. \\
& \left.+\left(\sigma_{p}^{(0)}-\sigma_{*}\right) \sin ^{2} t+\sigma_{p q}^{(0)} \sin (2 t)\right]
\end{aligned}
$$




$$
\begin{gathered}
\sigma_{p}(t)=\sigma_{*}+e^{-2 \gamma t}\left[\left(\sigma_{p}^{(0)}-\sigma_{*}\right) \cos ^{2} t\right. \\
\left.+\left(\sigma_{q}^{(0)}-\sigma_{*}\right) \sin ^{2} t-\sigma_{p q}^{(0)} \sin (2 t)\right] \\
\sigma_{q p}(t)=e^{-2 \gamma t}\left[\sigma_{q p}^{(0)} \cos (2 t)+\frac{1}{2}\left(\sigma_{p}^{(0)}-\sigma_{q}^{(0)}\right) \sin (2 t)\right]
\end{gathered}
$$

Any initial pure Gaussian state is unitarily equivalent to the squeezed state, defined as an eigenstate of the canonically transformed operator $\hat{b}=\cosh \rho \hat{a}+\sinh \rho \hat{a}^{\dagger}$ with a complex eigenvalue $\alpha \equiv \sqrt{a} \exp (i \phi)$ and a real 'squeezing parameter' $\rho$ (sometimes it is called also a 'two-photon state' 39]). Therefore we parametrize the initial variances and average values as

$$
\begin{gathered}
\sigma_{q}^{(0)}=\frac{1}{2} e^{-2 \rho}, \quad \sigma_{p}^{(0)}=\frac{1}{2} e^{2 \rho}, \quad \sigma_{p q}^{(0)}=0, \\
\bar{q}_{0}=\sqrt{2 a} e^{-\rho} \cos \phi, \quad \bar{p}_{0}=\sqrt{2 a} e^{\rho} \sin \phi .
\end{gathered}
$$

The 'total purity' $\mu$ does not depend on the first order moments of the coordinates:

$$
\mu=\left[(1+2 u \nu)^{2}+4 u(1-u)(1+2 \nu) \sinh ^{2} \rho\right]^{-1 / 2} .
$$

On the contrary, the 'diagonal purity' $\lambda$ depends on all 5 parameters of the one-dimensional Gaussian Wigner function.

The generic diagonal generating function found in [36, 40] can be expressed as

$$
G(z)=[\mathcal{G}(z)]^{-1 / 2} \exp \left(\frac{1}{D}\left[\frac{z g_{1}-z^{2} g_{2}}{\mathcal{G}(z)}-g_{0}\right]\right)
$$

where

$$
\begin{gathered}
\mathcal{G}(z)=\frac{1}{4}\left[(1+z)^{2}+4 d(1-z)^{2}+2\left(\sigma_{q}+\sigma_{p}\right)\left(1-z^{2}\right)\right] \\
D=1+2\left(\sigma_{p}+\sigma_{q}\right)+4 d \\
g_{0}=\bar{p}^{2}\left(2 \sigma_{q}+1\right)+\bar{q}^{2}\left(2 \sigma_{p}+1\right)-4 \bar{p} \bar{q} \sigma_{p q} \\
g_{1}=2 \bar{p}^{2}\left[\sigma_{q}^{2}+\sigma_{p q}^{2}+\sigma_{q}+\frac{1}{4}\right]+2 \bar{q}^{2}\left[\sigma_{p}^{2}+\sigma_{p q}^{2}+\sigma_{p}+\frac{1}{4}\right] \\
-4 \bar{p} \bar{q} \sigma_{p q}\left(\sigma_{q}+\sigma_{p}+1\right) \\
g_{2}=2 \bar{p}^{2}\left(\sigma_{q}^{2}+\sigma_{p q}^{2}-\frac{1}{4}\right)+2 \bar{q}^{2}\left(\sigma_{p}^{2}+\sigma_{p q}^{2}-\frac{1}{4}\right) \\
-4 \bar{p} \bar{q} \sigma_{p q}\left(\sigma_{q}+\sigma_{p}\right)
\end{gathered}
$$

For the initial pure squeezed states $G(z, t)$ can be written as

$$
\begin{aligned}
& G(z ; t)=\left(f-b z+c z^{2}\right)^{-1 / 2} \\
& \times \exp \left[-a(1-u) \frac{F-B z+C z^{2}}{f-b z+c z^{2}}\right]
\end{aligned}
$$


where

$$
\begin{gathered}
f=(1+u \nu)^{2}+(1-u)(1+u+2 u \nu) \sinh ^{2} \rho, \\
b=2 u \nu(1+u \nu)+2 u(1+2 \nu)(1-u) \sinh ^{2} \rho, \\
c=(u \nu)^{2}-(1-u)(1-u-2 u \nu) \sinh ^{2} \rho, \\
F=\frac{1}{2}[1-u+R(1+u+2 u \nu)] \\
B=1-u+R u(1+2 \nu) \\
C=\frac{1}{2}[1-u-R(1-u-2 u \nu)] \\
R=\cosh (2 \rho)-\sinh (2 \rho) \cos (2 \phi)
\end{gathered}
$$

Using formula (7) and the relations

$$
f-b+c=1, \quad F-B+C=0
$$

we obtain after some algebra the following integral representation for the time dependent 'diagonal purity' of a generic initially squeezed state:

$$
\lambda=\int_{0}^{\frac{\pi}{2}} \frac{2 d \gamma}{\pi \sqrt{\Phi(\gamma)}} \exp \left[-4 a(1-u)\left(V+Y \sin ^{2} \gamma\right) \frac{\sin ^{2} \gamma}{\Phi(\gamma)}\right]
$$

where

$$
\begin{gathered}
\Phi(\gamma)=\left(1+2 b \sin ^{2} \gamma\right)^{2}+(1-u)^{2} \sinh ^{2}(2 \rho) \sin ^{2}(2 \gamma) \\
V=R(1+2 u \nu)+(1-u)\left(R-1+4 R \sinh ^{2} \rho\right) \\
Y=4 u B\left[\nu(1+u \nu)+(1-u)(1+2 \nu) \sinh ^{2} \rho\right] \\
+2(1-u)\left(1-R-2 R \sinh ^{2} \rho\right)
\end{gathered}
$$

The evolution of the 'purity' and the 'coherence coefficient' for the highly squeezed $(\rho>1)$ initial state is illustrated in figure (5). In this case $\mu \approx \mathcal{C}$ up to the values of the dimensionless time $\tau=2 \gamma t \sim 1$. In contradistinction to the cases of coherent or 'cat' states, the coherence coefficient is not monotonous function of time, but it tries to follow the increase of the 'purity' at $\tau>1$, before going finally to zero.

The integral (54) can be easily calculated in the long-time limit $1-u=\exp (-2 \gamma t) \equiv \varepsilon \ll 1$ at zero temperature $(\nu=0): \lambda=1-2 \varepsilon\left(\sinh ^{2} \rho+a R\right)+\mathcal{O}\left(\varepsilon^{2}\right)$. Comparing this expression with the similar expansion of the 'total purity' $\mu$ (51) we obtain $\mathcal{C} \approx \mu-\lambda \approx 2 \varepsilon a R=2 \varepsilon \mathcal{E}_{c l}$, where

$$
\mathcal{E}_{c l} \equiv \frac{1}{2}\left(\bar{q}_{0}^{2}+\bar{p}_{0}^{2}\right)=a R
$$

is the initial 'classical' energy (the total energy without the contribution of the vacuum fluctuations). Then equation (29) yields the 'ultimate decoherence time' $t_{d}^{(s q)} \sim(2 \gamma)^{-1} \ln \left(2 \mathcal{E}_{c l} / \beta\right)$ which has the same order of magnitude as the time $t_{d}^{(c o h)}(\overline{30})$ for the coherent state with the same 'classical energy' $|\alpha|^{2}$ (at zero temperature $\mu_{e q}=1$ ). If $a=0$ (the initial squeezed vacuum 
state), then one should calculate $\mu$ and $\lambda$ up to the second order terms with respect to $\varepsilon$. In this case we obtain $\mathcal{C} \approx \mu-\lambda \approx \frac{1}{4} \varepsilon^{2} \sinh ^{2}(2 \rho)$, and $t_{d}^{(s q)} \sim(2 \gamma)^{-1} \ln [\sinh (2 \rho) / 2 \sqrt{\beta}]$. Since we consider the case $\rho \gg 1$, we can replace $\sinh (2 \rho) / 2$ by $\sinh ^{2}(\rho)=E-\frac{1}{2} \approx E$, where $E$ is the total energy in the case discussed.

\section{Thermalization dynamics}

The concrete evolution of the thermalization coefficient depends on a peculiar 'competition' between the 'total purity' $\mu(u)$ (which was calculated in the preceding sections) and the ground state probability $p_{0}(u)$, which can can be easily found from the 'diagonal generating function'

$$
p_{0}(t)=\frac{1}{1+\nu u(t)} G_{0}\left(\frac{u(t)(1+\nu)}{1+\nu u(t)}\right) .
$$

In the case of initial coherent state we have $\left(a \equiv|\alpha|^{2}\right) G_{0}^{(c o h)}(z)=\exp [a(z-1)]$. Consequently,

$$
p_{0}^{(c o h)}=\frac{1}{1+u \nu} \exp \left[-\frac{a(1-u)}{1+u \nu}\right]
$$

so the 'thermalization coefficient' reads

$$
\mathcal{D}_{a}^{(c o h)}(u)=\left\{1+\frac{1+2 u \nu}{(u \nu)^{2}}\left(1-\exp \left[-\frac{a(1-u)}{1+u \nu}\right]\right)\right\}^{-1 / 2}
$$

Evidently, the case $\nu=0$ should be excluded in this expression. The dependence on the displacement parameter $a$ disappears for $a(1-u) \gg 1$, when all the functions $\mathcal{D}_{a}^{(c o h)}(u)$ merge to $\mathcal{D}_{\infty}^{(c o h)}(u) \approx u \nu /(1+u \nu)$. This slow evolution is transformed into a fast transition to the equilibrium value if $a(1-u) \ll 1$ :

$$
\mathcal{D}_{a}^{(c o h)}(1-\varepsilon) \approx 1-\frac{1+2 \nu}{2(1+\nu)} \frac{a \varepsilon}{\nu^{2}}+\mathcal{O}\left(\varepsilon^{2}\right) .
$$

For the 'cat' states equations (23) and (36) yield

$$
\begin{aligned}
& p_{0}^{(c a t)}=\frac{2 \mathcal{N}^{2}}{1+u \nu} \exp \left[-\frac{a(1-u)}{1+u \nu}\right] \\
& \times\left\{1+\cos \varphi \exp \left[-\frac{2 a u(1+\nu)}{1+u \nu}\right]\right\} .
\end{aligned}
$$

At low temperatures $(\nu \ll 1)$ all the exponential functions 'die out' if $a(1-u) /(1+\nu) \gg 1$ and $a u /(1+\nu) \gg 1$, and we observe the 'plateau' $\mathcal{D}^{(c a t)}(u) \approx \frac{1}{3}$ (see figure 6).

For the squeezed state, formula (53) yields

$$
p_{0}^{(s q z)}=G(0, t)=f^{-1 / 2} \exp [-a(1-u) F / f] .
$$

For large values of the squeezing parameter $\rho$ and $\nu \ll 1$, the 'purity' (which does not depend on $a$ ) is given by $\mu \approx(2 \sinh \rho \sqrt{u(1-u)})^{-1} \ll 1$, unless $u$ is close enough to 0 or 1 . If 
$a(1-u) \gg 1$, then $p_{0} \ll 1$, and we observe a universal (independent of $\alpha$ ) behavior of the thermalization coefficient

$$
\mathcal{D}_{\infty}^{(s q)}(u) \approx \frac{1-\mu}{1+\mu} \approx \frac{4 u(1-u) \sinh ^{2} \rho}{\left(\sqrt{1+4 u(1-u) \sinh ^{2} \rho}+1\right)^{2}}
$$

The $\mathcal{D}$-factor rapidly increases for a small time interval $t<t_{1} \sim\left(\gamma \sinh ^{2} \rho\right)^{-1}$ (note that $\sinh ^{2} \rho$ is just the 'reduced' energy of fluctuations in the initial squeezed state, $\left.\mathcal{E}_{0}-\frac{1}{2}\right)$. For $t>t_{1}$ we observe some 'plateau', whose extension corresponds approximately to the interval $0.07<u<0.93$ (inside this interval, the values of the function $u(1-u)$ are not less than a half of the maximum value at $u=0.5)$. For the values of $u$ close to 1 , the $\mathcal{D}$-factor may decrease, following the decreasing linear entropy, but finally the term $p_{0}$ enters the game and prevents the thermalization coefficient from falling down to zero. This final stage of evolution seems very fast in terms of the 'compact time' $u$, but it is not so dramatic with respect to the scaled time $\tau=2 \gamma t$ : see figure 7 .

It is interesting to consider also the thermalization of the initial $M$-photon Fock state $|M\rangle$. In this case the off-diagonal elements of the statistical operator in the Fock basis are equal to zero identically for any time $t \geq 0$, so the 'total purity' $\mu$ coincides with the diagonal one $\lambda$. The initial diagonal generating function equals $G_{0}(z)=z^{M}$. Consequently,

$$
p_{0}(t)=\frac{[u(t)(1+\nu)]^{M}}{[1+\nu u(t)]^{M+1}},
$$

whereas the integral (17) can be transformed to the form

$$
\begin{gathered}
\lambda(t)=\int_{0}^{2 \pi} \frac{d \varphi}{2 \pi} \frac{[a+b \cos \varphi]^{M}}{[c-d \cos \varphi]^{M+1}} \\
a=u^{2}(1+\nu)^{2}+(1-u-u \nu)^{2}, \quad d=2 u \nu(1+u \nu) \\
b=2 u(1-u-u \nu), \quad c=\nu^{2} u^{2}+(1+u \nu)^{2}
\end{gathered}
$$

To calculate the integral (61) we designate it as $I_{M}$ and introduce a new generating function

$$
Q(y)=\sum_{n=0}^{\infty} I_{n} y^{n}=\int_{0}^{2 \pi} \frac{d \varphi}{2 \pi[c-y a-(d+y b) \cos \varphi]}
$$

The last integral is given by the expression [34

$$
Q(y)=\left[(c-y a)^{2}-(d+y b)^{2}\right]^{-1 / 2}
$$

which has the same structure as the known generating function of the Legendre polynomials, so after some algebra we obtain

$$
\lambda=\frac{|1-2 u(1+\nu)|^{M}}{(1+2 u \nu)^{M+1}} P_{M}\left(\frac{(1-u)^{2}+u^{2}(1+2 \nu)^{2}}{(1+2 u \nu)|1-2 u(1+\nu)|}\right)
$$

The typical dependences $\mathcal{D}(u)$ for the coherent and Fock states with different initial energies are given in figure 8 . 


\section{Three stages of decoherence and thermalization}

We see that at low temperatures the decoherence and thermalization of highly excited initial states go through three distinct stages. The first one is rather short, its characteristic time being determined completely by the initial energy of quantum fluctuations, $t_{1} \sim(\gamma \mathcal{E})^{-1}$. However, the coefficients $\mathcal{C}$ and $\mathcal{D}$ do not assume their equilibrium values ( 0 and 1 , respectively) at the end of this stage, but they remain approximately constant for a rather long period of time. The total destruction of coherence is observed only after the time $t_{d} \sim(2 \gamma)^{-1} \ln (E) \gg t_{1}$, where $E$ is either the total energy or its 'classical' part (depending on the initial state). This time tends to a finite limit when the temperature $T$ goes to zero.

The disappearance of the off-diagonal matrix elements of the statistical operator (decoherence) does not mean that the energy level populations reach their equilibrium values. This happens only after the 'thermalization time' $t_{T}$, which can be evaluated from the asymptotical behavior of the thermalization parameter $\mathcal{D}$ at $t \rightarrow \infty$ in the form of the Taylor expansion with respect to the small variable $\varepsilon=1-u=\exp (-2 \gamma t)$. For example, for a generic squeezed state with nonzero mean values of the quadrature components we have (see also (58))

$$
\mathcal{D}(1-\varepsilon)=1-\frac{(1+2 \nu) \mathcal{E}_{c l}}{2 \nu^{2}(1+\nu)} \varepsilon+\mathcal{O}\left(\varepsilon^{2}\right)
$$

where the 'classical energy' is given by (55)). Assuming (for $\nu \ll 1)\left(\mathcal{E}_{c l} / \nu^{2}\right) \exp (-2 \gamma t) \sim 1$ we obtain the estimation $t_{T} \sim(2 \gamma)^{-1} \ln \left(\mathcal{E}_{c l} / \nu^{2}\right)$ which shows that the 'thermalization time' may exceed essentially not only the decay time $\gamma^{-1}$ but the 'ultimate decoherence time' $t_{d}$, too. In particular, for the initial coherent state $(\rho=0)$ we have $t_{T} \sim \gamma^{-1} \ln |\alpha / \nu|$.

The situation resembles the classical theory of magnetic relaxation, where we have also two characteristic times: the time of transverse relaxation (dephasing) $T_{2}$ (analog of $t_{d}$ ) and the time of longitudinal relaxation $T_{1}$ (analog of $t_{T}$ ). The difference is that in our case both times depend not only on the properties of the environment (through the constants $\gamma$ and $\nu$ ), but also on the initial state (through its energy). Besides, there exists the third time - the 'primary decoherence time' $t_{1}$.

For the states with zero mean values of the quadratures, the expansion of $1-\mathcal{D}$ begins with the quadratic term $\varepsilon^{2}=\exp (-4 \gamma t)$. For the initial vacuum squeezed state $(\alpha=0)$ we have

$$
\mathcal{D}=1-\left[\frac{\sinh (2 \rho)}{4 \nu(1+\nu)} \varepsilon\right]^{2}+\mathcal{O}\left(\varepsilon^{3}\right) .
$$

If $\rho>1$ and $\nu \ll 1$, one can rewrite $(63)$ as

$$
\mathcal{D} \approx 1-[E \varepsilon /(2 \nu)]^{2}
$$

where $E=\sinh ^{2} \rho+\frac{1}{2} \approx \frac{1}{4} \exp (2 \rho)$ is the total energy of the initial state (it coincides with the energy of fluctuations in the case involved). Consequently, $t_{T}^{s q-v a c} \sim(2 \gamma)^{-1} \ln (E / \nu)$.

A similar behavior of $\mathcal{D}(u)$ at $1-u \ll 1$ is observed for the 'cat' states. If $a \gg 1$, then the dependence on the phase $\varphi$ becomes unimportant, and we obtain

$$
\mathcal{D}=1-\frac{(a \varepsilon)^{2}(1+2 \nu)}{4 \nu^{2}(1+\nu)^{2}}+\mathcal{O}\left(\varepsilon^{3}\right) .
$$


In this case the total energy $E \approx a \gg 1$, and we arrive again at the equation (64) (if $\nu \ll 1$ ) which yields $t_{T}^{\text {cat }} \sim(2 \gamma)^{-1} \ln (E / \nu)$, similarly to the case of the vacuum squeezed state.

For the Fock states we obtain

$$
\mathcal{D}(1-\varepsilon)=1-\frac{M(M+1)}{4 \nu(1+\nu)^{2}} \varepsilon^{2}+\cdots .
$$

If $M \gg 1$, then $E \approx M$, and we have $t_{T}^{\text {Fock }} \sim \gamma^{-1} \ln (E / \sqrt{\nu})$. We see that the 'thermalization time' $t_{T}$ depends logarithmically on the initial energy. Besides, it has the strong temperature dependence, growing as $T^{-1}$ at $T \rightarrow 0$ (remember that $\nu=\left[\exp \left(\hbar \omega / k_{B} T\right)-1\right]^{-1}$, so $\nu \approx \exp \left(-\hbar \omega / k_{B} T\right)$ at $\left.T \rightarrow 0\right)$, in a complete agreement with the third law of thermodynamics. The dependence of the 'thermalization time' on the mean equilibrium photon number $\nu$ enables ordering different families of quantum states with respect to their robustness against the thermalization (while the 'primary time' $t_{1}$ is the same for all states with equal values of the energy of quantum fluctuations). The coherent states are the most robust ones, then follow squeezed and 'cat' states, whereas the Fock states, being 'the most unclassical states', are thermalized much faster than all the others.

Another interesting feature of the decoherence and thermalization process is the existence of 'plateaus' in the dependences $\mathcal{C}(u)$ and $\mathcal{D}(u)$ for several different types of states (excluding the coherent states) possessing high initial energy (provided the temperature is low enough). In the cases of the squeezed and Fock states the altitudes of 'plateaus' tend to 1 for $\mathcal{D}(u)$ and to 0 for $\mathcal{C}(u)$ when the initial energy increases. But for the coherent 'cat' states, the metastable values of the coherence and thermalization coefficients remain finite even for $E \rightarrow \infty: \mathcal{C}_{p l t} \sim \frac{1}{2}$ and $\mathcal{D}_{p l t} \sim \frac{1}{3}$. Consequently, some degree of coherence (with respect to the fixed energy basis) survives in the 'cat' states for a long period of time $t<t_{d} \sim(2 \gamma)^{-1} \ln a$. Perhaps, this fact could be important for applications.

\section{Conclusion}

We may conclude that the new quantitative measures of decoherence and thermalization shed new light on the details of the decoherence process accompanying the 'standard' thermal relaxation of a quantum harmonic oscillator, showing that this process has three distinct stages in the case of highly excited initial pure states and low temperatures. In particular, we have shown that at low temperatures the 'ultimate decoherence' is achieved after rather long interval of time, which is essentially greater than the relaxation time and the "primary decoherence time' which was the central subject of previous studies. Our analysis permits to find the conditions under which the 'purity' or the 'linear entropy' can serve as reasonable measures of (de)coherence: the initial energy of the quantum state must be much greater then the mean energy of the reservoir oscillators, $E_{0} \gg 1+2 \nu$. However, even under this condition the 'purity' can be used only to describe the initial stage of the relaxation process, but it cannot replace the true measures of 'coherence' for the whole time interval. 


\section{Acknowledgements}

ALS thanks CAPES (Brasil) for support. SSM thanks CNPq (Brasil) for partial financial support.

\section{A Propagator of the Fokker-Planck equation}

Under certain conditions the process of relaxation of linear $r$-dimensional quantum systems (such as a system of coupled oscillators or a charged particle in a homogeneous electromagnetic field and in a confining parabolic potential) can be described in the framework of the FokkerPlanck equation for the Wigner function [29, 42]-45]

$$
\frac{\partial W}{\partial t}=-\frac{\partial}{\partial y_{i}}\left[(\mathbf{A y}+\mathbf{K})_{i} W\right]+D_{i j} \frac{\partial^{2} W}{\partial y_{i} \partial y_{j}}
$$

where $i, j=1,2, \ldots, 2 r$; the $2 r$-dimensional vector $\mathbf{y}$ consists of the linear combinations of the Cartesian coordinates $q_{i}$ and canonically conjugated momenta $p_{i}$ (in the simplest case $\mathbf{y}=(\mathbf{q}, \mathbf{p}))$. The drift matrix $\mathbf{A}$ and vector $\mathbf{K}$ do not depend on the phase space vector variable $\mathbf{y}$, although they may have, in general, arbitrary dependences on time. However, the diffusion symmetric matrix $\mathbf{D} \equiv\left\|D_{i j}\right\|$ cannot be arbitrary, since the physically acceptable solutions to equation (A.1) must satisfy the condition of the positive semidefiniteness of the corresponding statistical operator. This condition is fulfilled provided the matrix $\mathbf{D}_{*}=\mathbf{D}+\frac{i \hbar}{4}(\mathbf{A} \Sigma+\Sigma \tilde{\mathbf{A}})$ is positively semidefinite [29, 42, 43]. The elements of the antisymmetric c-number matrix $\Sigma=\left\|\Sigma_{j k}\right\|$ are the commutators $\Sigma_{j k}=\frac{i}{\hbar}\left[\hat{y}_{j}, \hat{y}_{k}\right]$. In the case of the single space coordinate the matrix condition $\mathbf{D}_{*} \geq 0$ is equivalent to three scalar conditions [46, 47, 48, 49]

$$
\begin{gathered}
D_{p p} D_{q q}-D_{p q}^{2} \equiv \operatorname{det} \mathbf{D} \geq \frac{\hbar^{2}}{16}(\operatorname{Tr} A)^{2} \\
D_{p p} \geq 0, \quad D_{q q} \geq 0, \quad \mathbf{D}=\left\|\begin{array}{cc}
D_{p p} & D_{p q} \\
D_{p q} & D_{q q}
\end{array}\right\| .
\end{gathered}
$$

Since (A.1) can be considered as the Schrödinger equation with an effective quadratic (although non-Hermitian) Hamiltonian, the propagator $G\left(\mathbf{y}, \mathbf{y}^{\prime}, t\right)$,

$$
W(\mathbf{y}, t)=\int d \mathbf{y}^{\prime} G\left(\mathbf{y}, \mathbf{y}^{\prime}, t\right) W\left(\mathbf{y}^{\prime}, 0\right),
$$

can be found with the aid of the method of quantum time-dependent invariants given in [30, 50, 51]. However, to find its explicit expression it is sufficient to know that this propagator is a Gaussian, so, as any Gaussian Wigner function [29, 30, 52, 53] it can be written as

$$
\begin{aligned}
& G\left(\mathbf{y}, \mathbf{y}^{\prime}, t\right)=(2 \pi)^{-r}\left[\operatorname{det} \mathcal{M}_{*}(t)\right]^{-1 / 2} \\
& \times \exp \left\{-\frac{1}{2}\left[\mathbf{y}-\mathbf{y}_{*}(t)\right] \mathcal{M}_{*}^{-1}\left[\mathbf{y}-\mathbf{y}_{*}(t)\right]\right\}
\end{aligned}
$$

where $\mathbf{y}_{*}\left(\mathbf{y}^{\prime}, t\right)$ is the mean value of the phase space vector $\mathbf{y}$ and $\mathcal{M}_{*}(t)$ is the variance matrix. The explicit form of $\mathbf{y}_{*}$ and $\mathcal{M}_{*}$ can be obtained by solving the equations (which are immediate consequences of the Fokker-Planck equation (A.1))

$$
\dot{\mathcal{M}}_{*}=\mathbf{A} \mathcal{M}_{*}+\mathcal{M}_{*} \widetilde{\mathbf{A}}+2 \mathbf{D}
$$




$$
\dot{\mathbf{y}}=\mathrm{Ay}+\mathrm{K}
$$

with the initial conditions $\mathcal{M}_{*}(0)=0$ and $\mathbf{y}_{*}\left(\mathbf{y}^{\prime}, 0\right)=\mathbf{y}^{\prime}$, which are equivalent to the property $G\left(\mathbf{y}, \mathbf{y}^{\prime}, 0\right)=\delta\left(\mathbf{y}-\mathbf{y}^{\prime}\right)$ distinguishing the propagator from all other Gaussians.

In the case under study equations (A.4) coincide with the set (42)-(45). Putting their solutions (46)-(50) to the right-hand side of (A.3) we obtain the propagator (16). 


\section{References}

[1] Schrödinger E 1935 Naturwissenschaften 23812

[2] Dodonov V V, Malkin I A and Man`ko V I 1974 Physica 72597

[3] Yurke B and Stoler D 1986 Phys. Rev. Lett. 5713

[4] Bužek V and Knight P L 1995 Progress in Optics vol 34, ed E Wolf (Amsterdam: Elsevier) p 1

[5] Brif C 1996 Ann. Phys. (NY) 251180

[6] Caldeira A O and Leggett A J 1985 Phys. Rev. A 311059

[7] Walls D F and Milburn G J 1985 Phys. Rev. A 312403

[8] Savage C M and Walls D F 1985 Phys. Rev. A 322316

[9] Bužek V, Vidiella-Barranco A and Knight P L 1992 Phys. Rev. A 456570

[10] Kim M S and Bužek V 1992 Phys. Rev. A 464239

[11] Zurek W H, Habib S and Paz J P 1993 Phys. Rev. Lett. 701187

[12] Coetsch P, Graham R and Haake F 1996 Quant. Semicl. Opt. 8157

[13] Davidovich L, Brune M, Raimond J M and Haroche S 1996 Phys. Rev. A 531295

[14] Kim J I, Nemes M C, de Toledo Piza A F R and Borges H E 1996 Phys. Rev. Lett. 77207

[15] Moussa M H Y, Mizrahi S S and Caldeira A O 1996 Phys. Lett. A 221145

[16] Mizrahi S S, Dodonov V V and Otero D 1994 Mod. Phys. Lett. B 8995

[17] Isar A, Sandulescu A and Scheid W 1999 Phys. Rev. E 606371

[18] Dodonov V V, Man'ko O V, Man'ko V I and Wünsche A 1999 Physica Scripta 5981

[19] Wigner E P 1932 Phys. Rev. 40749

[20] Hillery M, O'Connell R F, Scully M O and Wigner E P 1984 Phys.Rep. 106121

[21] Weidlich W and Haake F 1965 Z. Phys. 18530

[22] Scully M O and Lamb W E 1967 Phys. Rev. 159208

[23] Gallis M R 1996 Phys. Rev. A 53655

[24] Isar A 1999 Fortschr. Phys. 47855

[25] Wang M C and Uhlenbeck G E 1945 Rev. Mod. Phys. 17323 
[26] Agarwal G S 1971 Phys. Rev. A 4739

[27] Haken H 1975 Rev. Mod. Phys. 4767

[28] Schoendorff L and Risken H 1990 Phys. Rev. A 415147

[29] Dodonov V V and Man'ko V I 1987 Group Theory, Gravitation and Elementary Particle Physics, Proceedings of Lebedev Physics Institute vol 167, ed A A Komar (Commack: Nova Science) p 7

[30] Dodonov V V and Man'ko V I 1989 Invariants and Evolution of Nonstationary Quantum Systems, Proceedings of Lebedev Physics Institute vol 183, ed M A Markov (Commack: Nova Science) p 263

[31] Zel'dovich B Ya, Perelomov A M and Popov V S 1968 Zhurn. Eksp. Teor. Fiz. 55589 [1969 Sov. Phys. JETP 28 308]

[32] Schell A and Barakat R 1973 J. Phys. A: Math. Gen. 6826

[33] Arnoldus H F 1996 J. Opt. Soc. Am. B 131099

[34] Gradshtein I S and Ryzhik I M 1994 Tables of Integrals, Series and Products (New York: Academic)

[35] Schramm P and Grabert H 1986 Phys. Rev. A 344515

[36] Dodonov V V, Man'ko O V and Man'ko V I 1994 Phys. Rev. A 492993

[37] Schrödinger E 1930 Sitzungsber. K. Preuss. Akad. Wiss. Phys. Math. Kl. 24296 Robertson H R 1930 Phys. Rev. 35667

[38] Dodonov V V, Kurmyshev E V and Man'ko V I 1980 Phys. Lett. A 79150

[39] Yuen H P 1976 Phys. Rev. A 132226

[40] Dodonov V V and Man'ko V I 1994 J. Math. Phys. 354277

[41] Dodonov V, Mizrahi S and de Souza Silva A L 1999 Annals of the Brazilian Commission for Optics vol 1, eds M Muramatsu and C H Monken, p 23

[42] Dodonov V V and Man'ko O V 1985 Physica A 130353

[43] Dodonov V V and Man'ko V I 1988 Classical and Quantum Effects in Electrodynamics, Proceedings of Lebedev Physics Institute vol 176, ed A A Komar (Commack: Nova Science) p 53

[44] Isar A, Sandulescu A, Scutaru H, Stefanescu E and Scheid W 1994 Int. J. Mod. Phys. E 3635

[45] Dodonov V V, Man'ko O V and Man'ko V I 1995 J. Russian Laser Research 161 
[46] Dodonov V V and Man'ko V I 1985 Group Theoretical Methods in Physics, Proceedings of the Second International Seminar, Zvenigorod, 1982 vol 1, eds M A Markov, V I Man'ko and A E Shabad (New York: Harwood Academic Publ.) p 705

[47] Barchielli A 1983 Nuovo Cim. B 74113

[48] Dekker H and Valsakumar M C 1984 Phys. Lett. A 10467

[49] Sandulescu A and Scutaru H 1987 Ann. Phys. (N.Y.) 173277

[50] Dodonov V V, Malkin I A and Man'ko V I 1975 Int. J. Theor. Phys. 1437

[51] Dodonov V V and Man'ko V I 1978 Physica A 94403

[52] Dodonov V V, Man'ko O V and Man'ko V I 1994 Phys. Rev. A 50813

[53] Tegmark M and Shapiro H S 1994 Phys. Rev. E 502538 
Figure 1: The 'purity' $\mu$ and the 'coherence' $\mathcal{C}$ versus the 'compact time' $u \equiv 1-e^{-2 \gamma t}$ for the initial coherent state with $|\alpha|^{2}=1$ and for three diferent temperatures. The order of the curves from top to bottom (in the left-hand side of the figure): $\mathcal{C}$ for $\nu=0$ (zero temperature) (in this case $\mu \equiv 1) ; \mu$ for $\nu=1, \mathcal{C}$ for $\nu=1 ; \mu$ for $\nu=10, \mathcal{C}$ for $\nu=10$.

Figure 2: The 'purity' $\mu$ and the 'coherence' $\mathcal{C}$ versus the 'compact time' $u \equiv 1-e^{-2 \gamma t}$ for the initial coherent state with $|\alpha|^{2}=10$ and for three diferent temperatures. The order of the curves from top to bottom (in the left-hand side of the figure): $\mathcal{C}$ for $\nu=0$ (zero temperature) (in this case $\mu \equiv 1$ ); $\mu$ for $\nu=1, \mathcal{C}$ for $\nu=1 ; \mu$ for $\nu=10, \mathcal{C}$ for $\nu=10$.

Figure 3: The 'purity' $\mu$ and the 'coherence' $\mathcal{C}$ versus the 'compact time' $u \equiv 1-e^{-2 \gamma t}$ for the initial odd coherent state $(\varphi=\pi)$ for different values of the parameters $a \equiv|\alpha|^{2}$ and $\nu$. The order of the curves from top to bottom (in the right-hand side of the figure) is as follows. I $\mu$ for $a=1$ and $\nu=0$; II $-\mu$ for $a=10$ and $\nu=0$; III $-\mathcal{C}$ for $a=10$ and $\nu=0$; IV $-\mathcal{C}$ for $a=1$ and $\nu=0 ; \mathrm{V}-\mu$ for $a=2$ and $\nu=5 ; \mathrm{VI}-\mathcal{C}$ for $a=2$ and $\nu=5$.

Figure 4: The 'accompanying coherence' $\mathcal{F}$ versus the 'compact time' $u \equiv 1-e^{-2 \gamma t}$ for the initial even coherent state $(\varphi=0)$ with $|\alpha|^{2}=10$, for three different temperatures: $\nu=0,1,10$.

Figure 5: The 'purity' $\mu$ and the 'coherence' $\mathcal{C}$ versus the 'compact time' $u \equiv 1-e^{-2 \gamma t}$ for the initial squeezed coherent state with $\rho=3,|\alpha|^{2}=1$ and $\phi=\pi / 2$, for two values of the equilibrium mean photon number in the reservoir: $\nu=0$ (two close curves relatively far off the bottom) and $\nu=2$ (two close curves nearby the bottom). In each pair of close curves the upper one corresponds to $\mu$ while the lower one gives $\mathcal{C}$. The curves inside the internal box give the same functions versus the usual (scaled) time $\tau=2 \gamma t$.

Figure 6: The 'thermalization coefficient' $\mathcal{D}$ versus the 'compact time' $u \equiv 1-e^{-2 \gamma t}$ for the initial odd coherent state $(\varphi=\pi)$ with different values of the parameter $|\alpha|^{2}=1,20$; in the low temperature $(\nu=0.01)$ and high temperature $(\nu=10)$ cases.

Figure 7: The 'thermalization coefficient' $\mathcal{D}$ versus the 'compact time' $u \equiv 1-e^{-2 \gamma t}$ for the initial squeezed coherent state with $\rho=3,|\alpha|^{2}=1$ and $\phi=\pi / 2$, for two values of the equilibrium mean photon number in the reservoir: $\nu=0.01$ (lower curves) and $\nu=2$ (upper curves). The curves inside the internal box give the same functions versus the usual (scaled) time $\tau=2 \gamma t$. 
Figure 8: The 'thermalization coefficient' $\mathcal{D}$ versus the 'compact time' $u \equiv 1-e^{-2 \gamma t}$ for the initial coherent and Fock states with equal mean numbers of photons: $M=|\alpha|^{2}=1$ and $M=|\alpha|^{2}=20$, in the low temperature $(\nu=0.01)$ and high temperature $(\nu=10)$ cases. I the Fock state with $M=1$ and $\nu=10$, II - the Fock state with $M=20$ and $\nu=10$, III - the Fock state with $M=20$ and $\nu=0.01, \mathrm{IV}$ - the coherent state with $|\alpha|^{2}=20$ and $\nu=10, \mathrm{~V}-$ the Fock state with $M=1$ and $\nu=0.01$, VI - the coherent state with $|\alpha|^{2}=1$ and $\nu=0.01$, VII - the coherent state with $|\alpha|^{2}=20$ and $\nu=0.01$. The internal box shows the behaviour of the last two curves in another scale. 


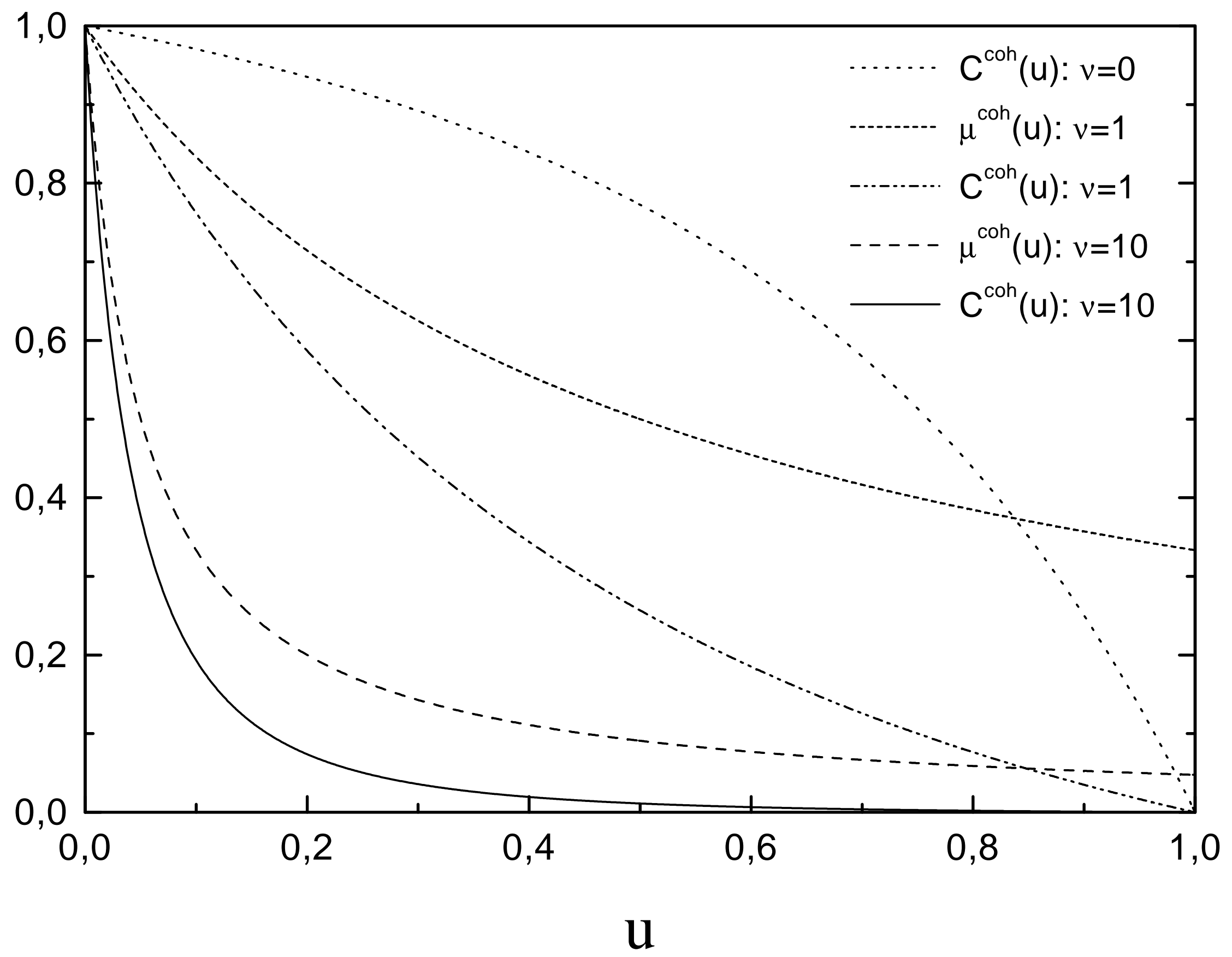




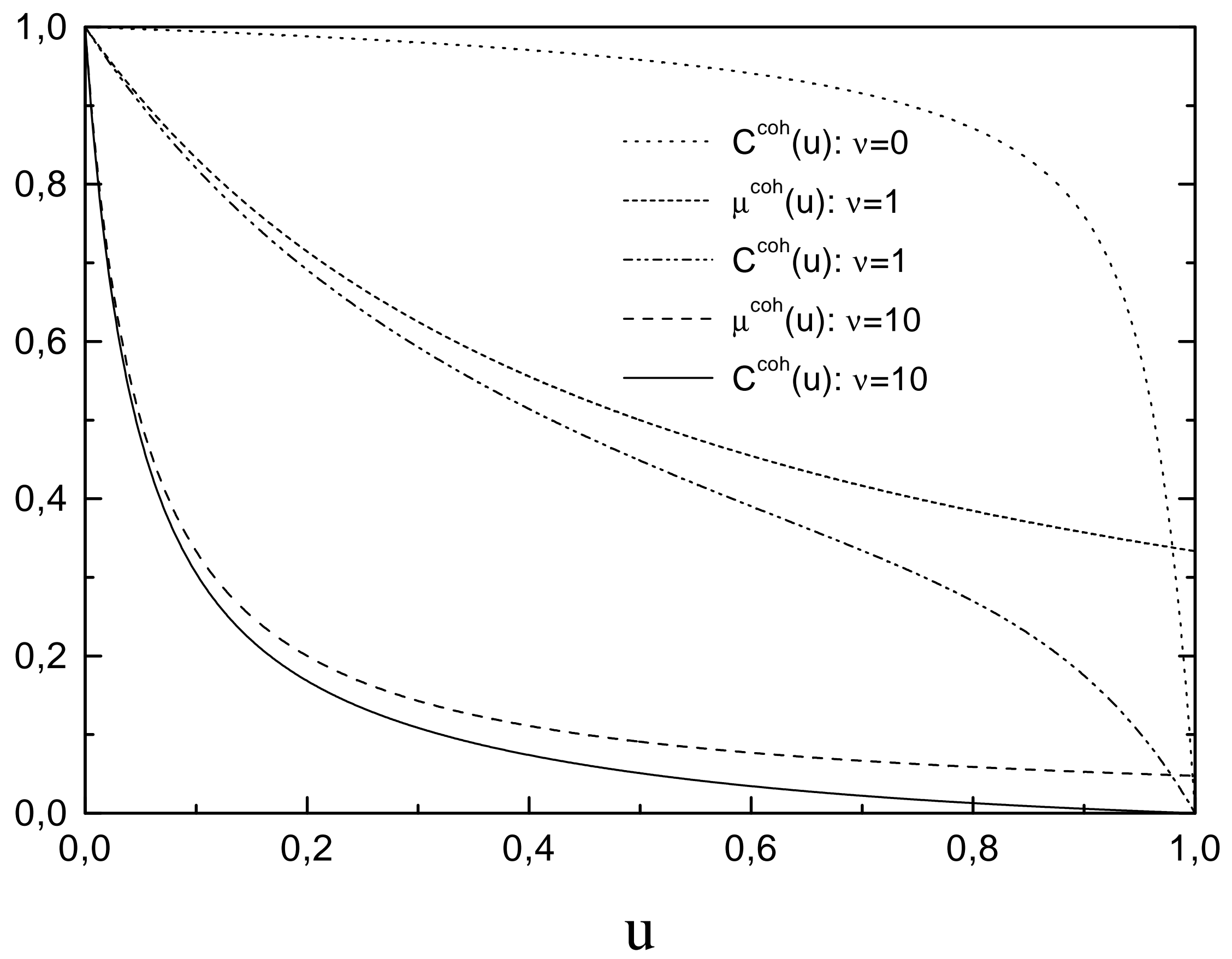




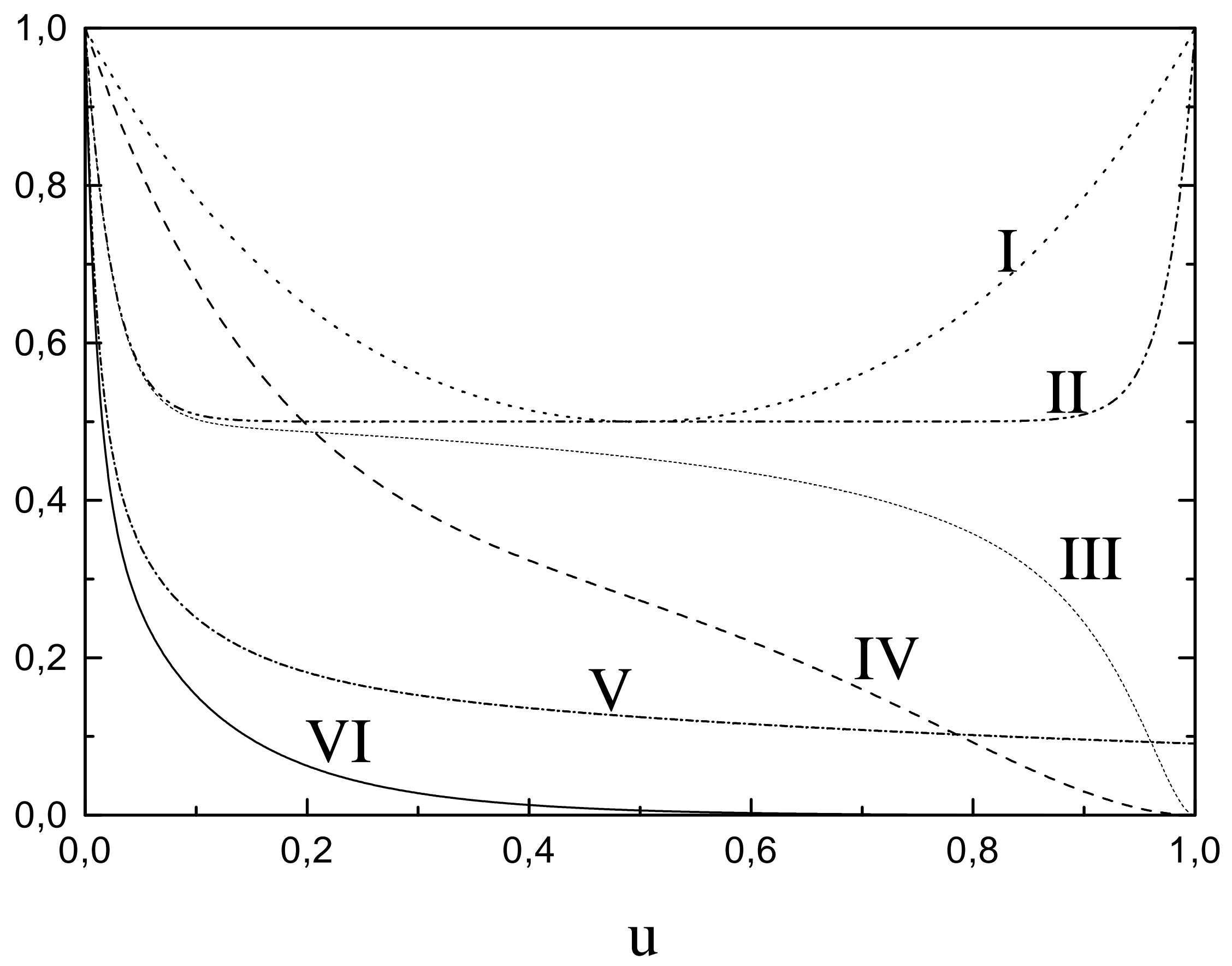




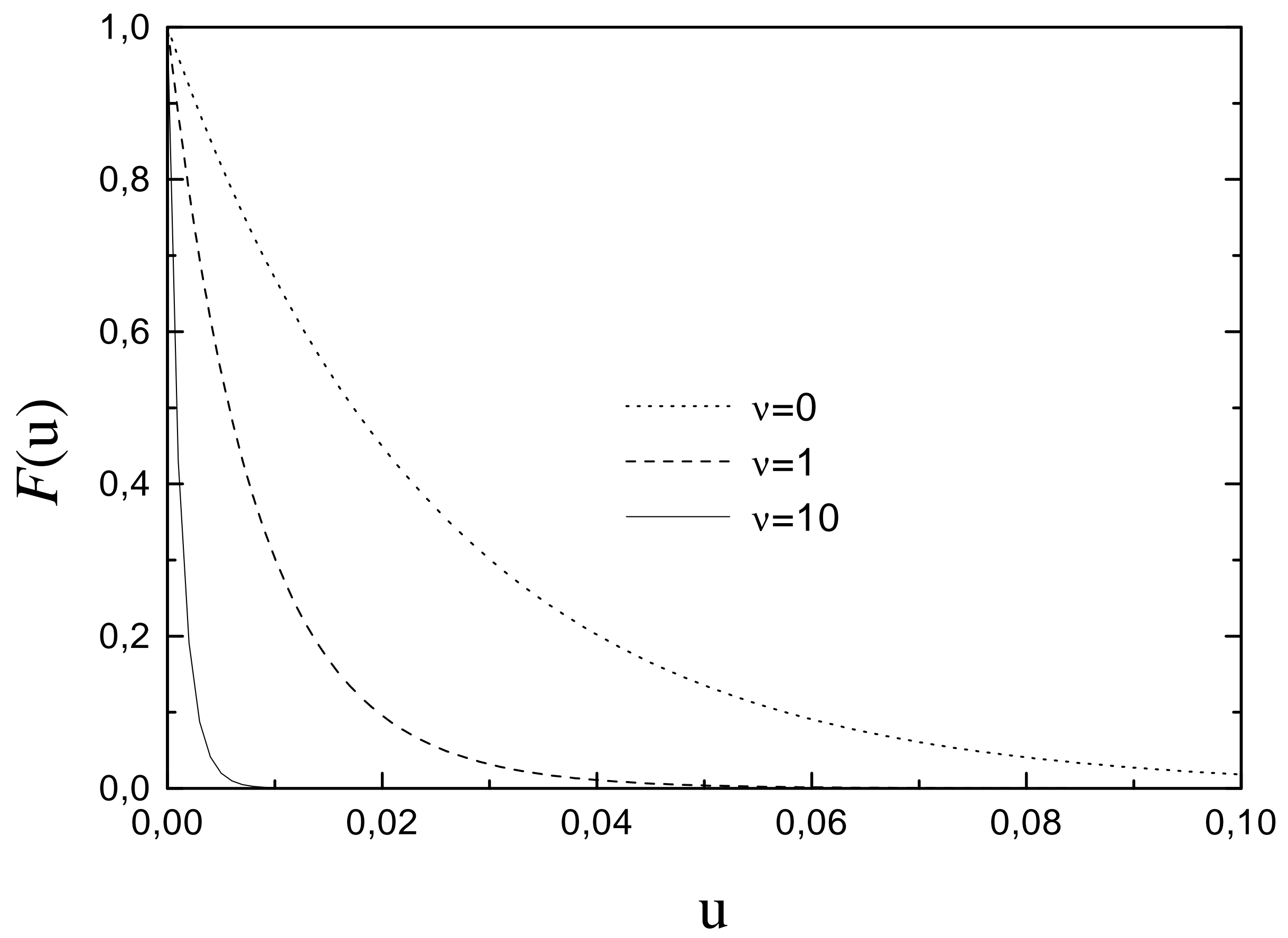




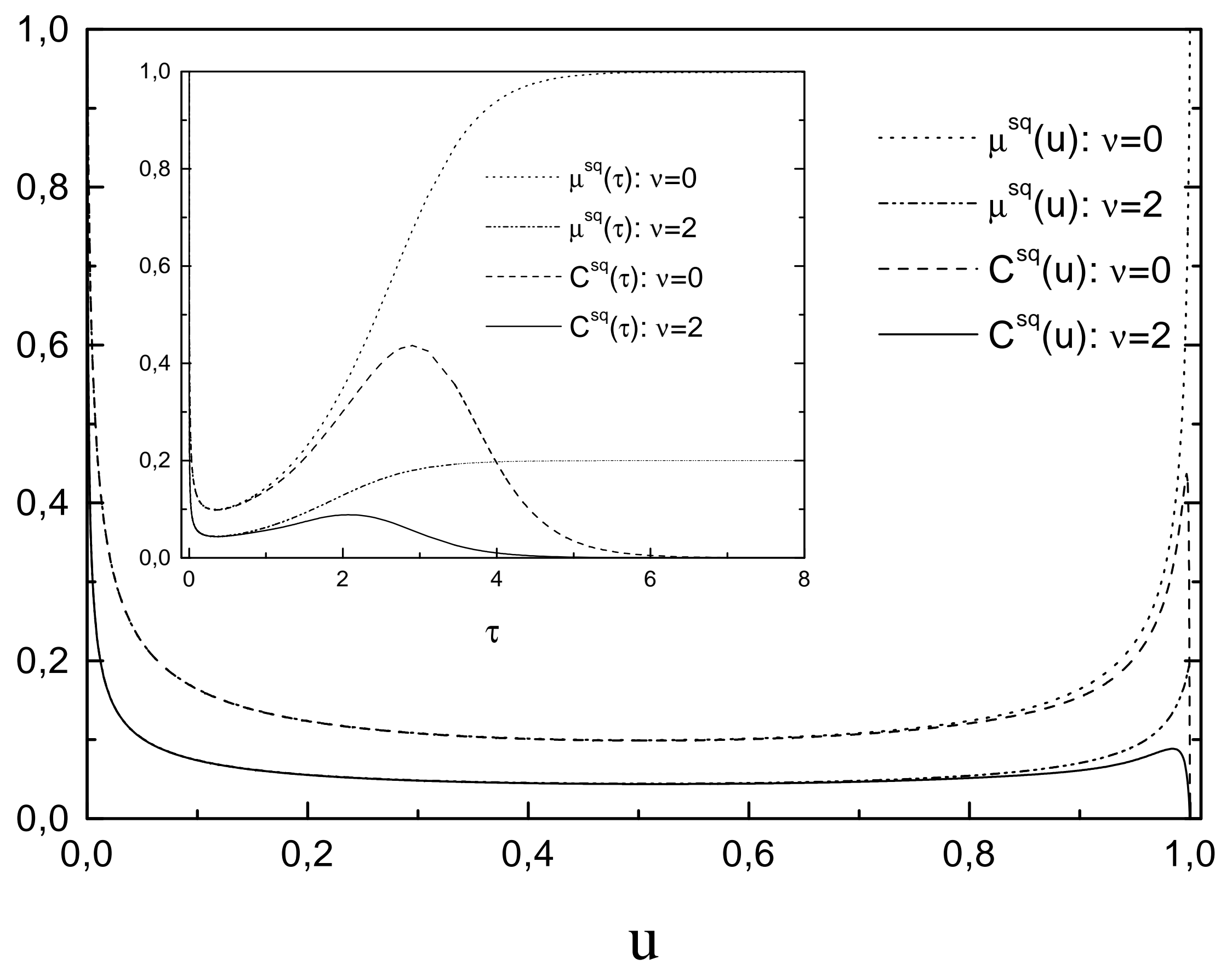




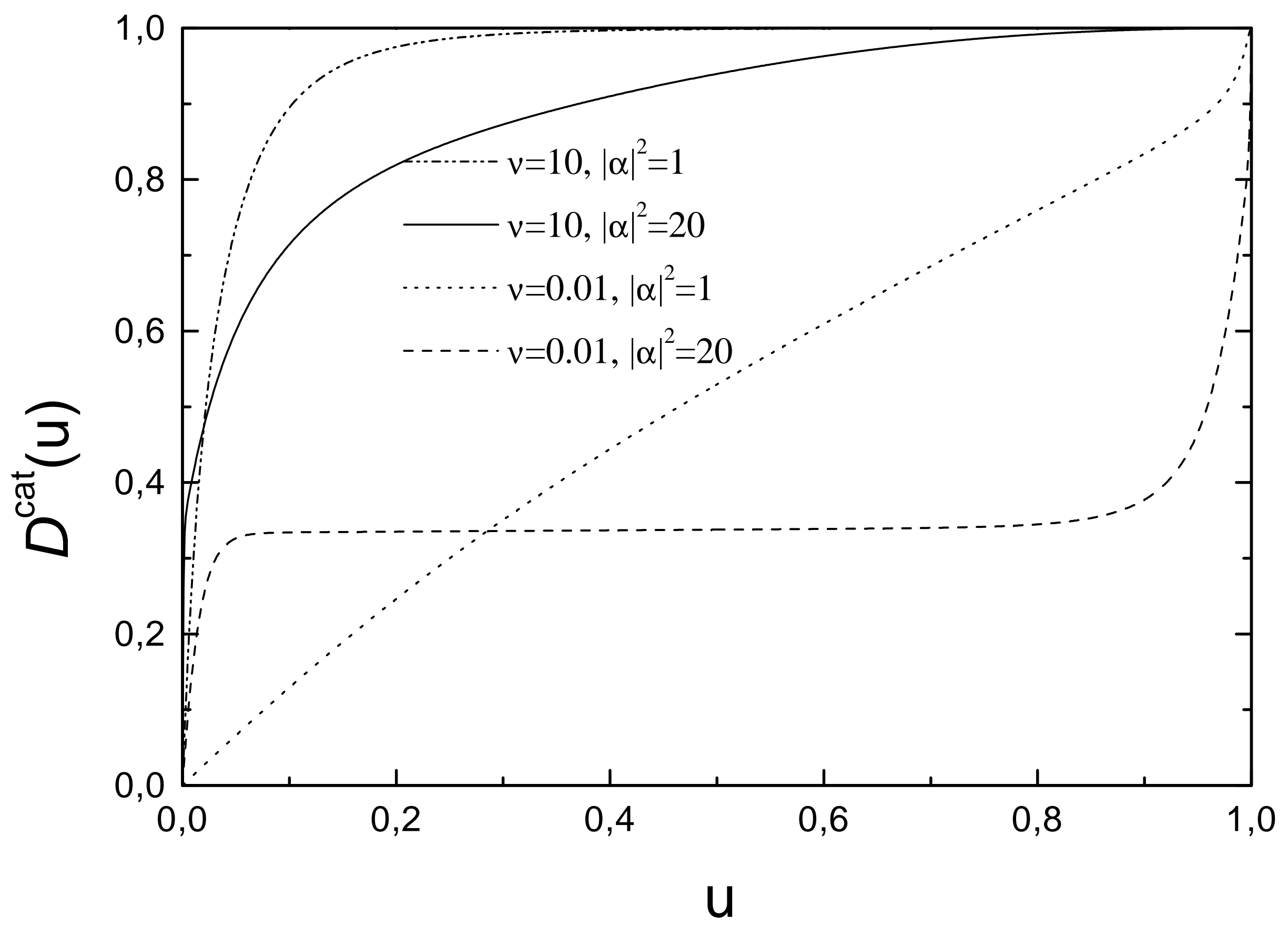




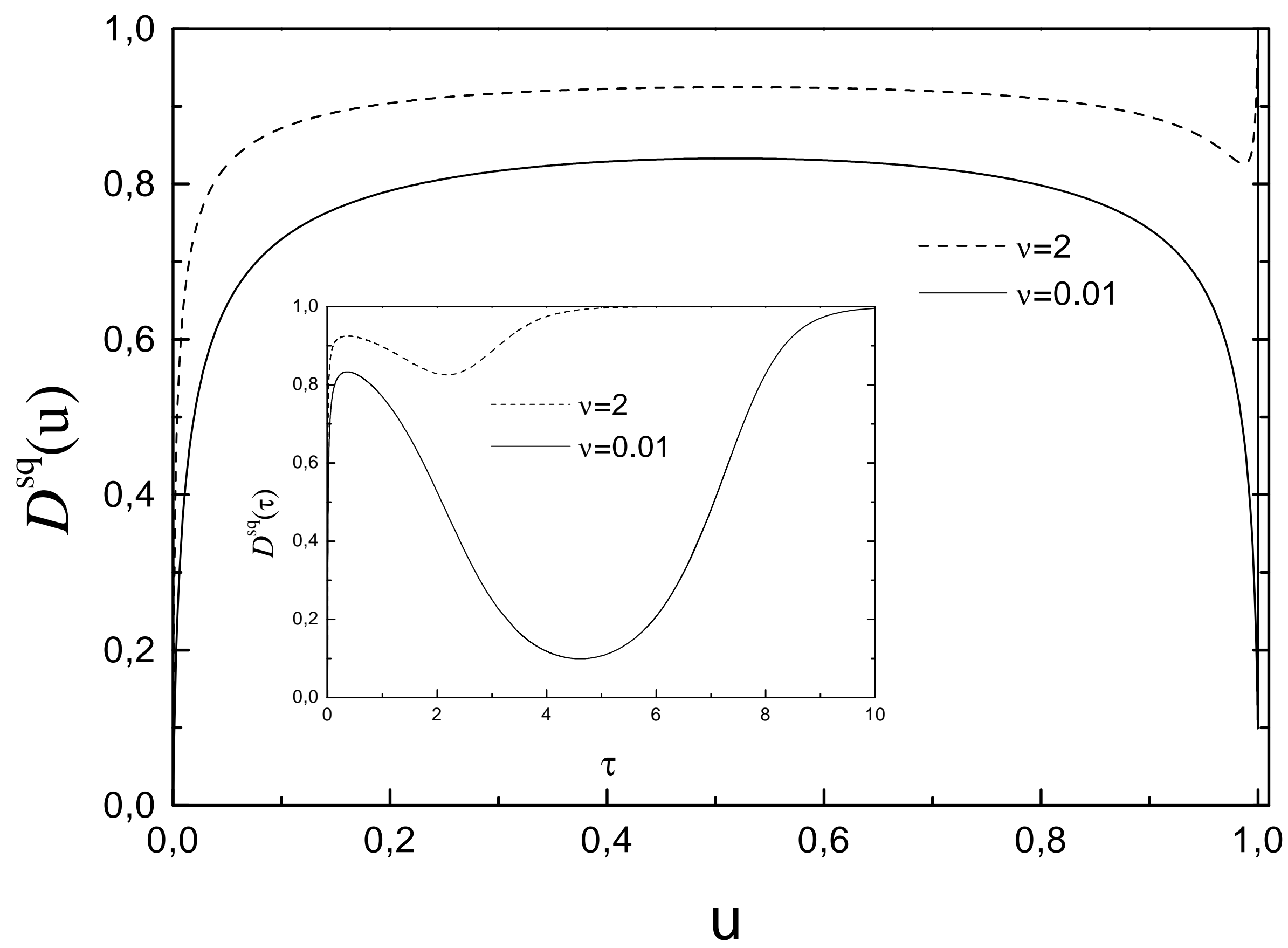




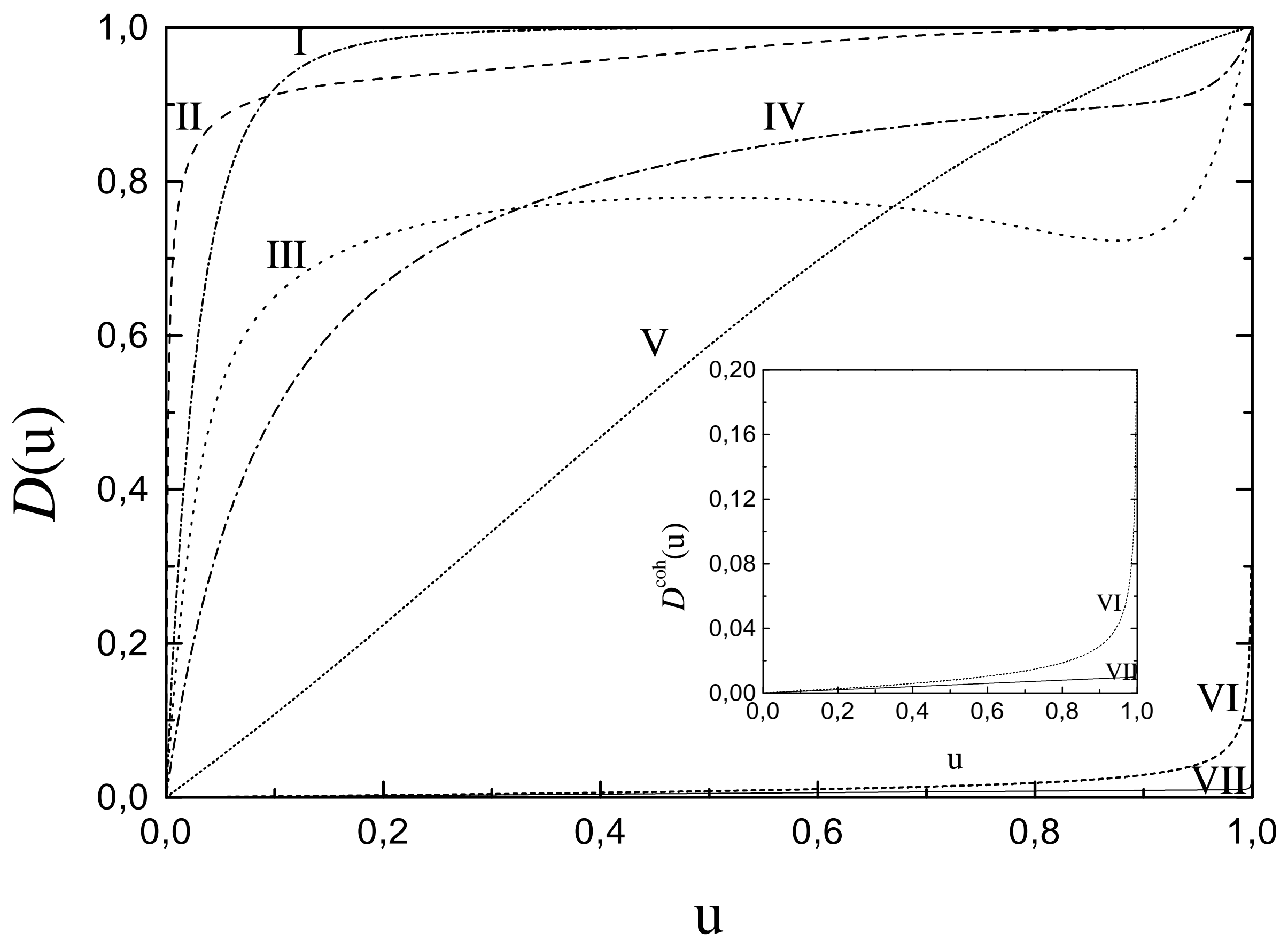

